\title{
5. The Nature of Knowledge and its Sharing through Models
}

\author{
Peter Bernus, ${ }^{1}$ Brane Kalpic ${ }^{2}$ \\ 1 Griffith University Email: P.Bernus@bigpond.com \\ 2 ETI Elektroelement Email: Brane.Kalpic@eti.si
}

\begin{abstract}
Enterprise Modelling has been repeatedly proposed as a way to share knowledge within and among companies. However, industry practitioners especially in Small and Medium Enterprises - are slow to take up this practice, and models are usually only built to support the development of application programs, databases or other information technology artefacts, rather then for the broader purpose of knowledge sharing.

The article examines knowledge categories previously proposed in the literature and propases an extension of previous work in order to better understand the nature of knowledge sharing processes and the role of models in these.
\end{abstract}

\section{INTRODUCTION}

In the literature, several different definitions of knowledge can be found. The Oxford English dictionary (1999) defines knowledge as the "facts, feelings, or experiences known by a person or group of people".

According to Baker et al (1997), knowledge is present in "ideas, judgements, talents, root causes, relationships, perspectives and concepts". Knowledge can be related to customers, products, processes, culture, skills, experiences and know-how.

Bender and Fish (2000) consider that knowledge originates in the head of an individual (the mental state of having ideas, facts, concepts, data and techniques, as recorded in an individual's memory) and is built on the basis of information transformed and enriched by personal experience, beliefs and values with decision and action-relevant meaning. Relevantly, therefore, knowledge formed by an individual could differ from knowledge possessed by another person receiving the same information.

Similarly to the above definition Baker et al (1997) define knowledge in the form of a simple formula:

\section{(1) Knowledge = Information $+[$ Skills + Experience + Personal Capability $]$}

This simple equation must be interpreted to give knowledge a deeper meaning: knowledge is created from data which becomes information as interpreted and remembered by a person with given skills, experience, personal capabilities and previously developed mental models.

Knowledge gives a person the ability to use information to guide the actions of the person in a manner that is appropriate to the situation. It is noteworthy that this does not imply that the person is aware of this knowledge or that he/she can explain (externalise) it. These distinctions are important to consider when planning to 
discover what knowledge is available, or intending to establish knowledge transfer/sharing.

Reading equation (1) it seems to suggest that knowledge equals the sum of the listed components. However, the intention is clearly to suggest that knowledge is an outcome of a process performed by an individual, i.e. it is a function of the listed components, which gives equation (2).

\section{(2) Knowledge $=$ f (Information, Skills, Previous Experience, Personal Capability)}

Still, equation (2) is not clear about the role of pre-existing knowledge in gaining new knowledge nor about the role of unlearning / transforming existing knowledge. Also neither equation explains what knowledge is - they only state that knowledge is created using these components. We would at least expect an equation that would have the pattern:

$$
\text { Knowledge } t_{t 2}=f\left(\text { Information } t_{<t-t 2>}, \ldots, \text { Knowledge } e_{t 1}, \ldots\right)
$$

Thus such an equation would explain how information gained between times $t 1$ and $\mathfrak{t} 2$ transforms knowledge, depending on many factors, including knowledge possessed before time $t 1$.

The authors believe that without improving the understanding of the nature of knowledge it would be difficult to pinpoint the role of models in gaining, capturing or sharing knowledge. Therefore this article sets out to investigate categories of knowledge (Section 2) and then identifies processes (Section 3) that transform knowledge in one category to knowledge in another category. Once such processes have been identified it is possible to identify those which can (or could) use models.

Note that the word 'models' here refers to a mathematical construct that can be used to represent a significant set of properties of some existing or proposed artefact, such that all relevant properties of the artefact can be derived by investigating the model rather then the artefact itself and no relevant properties can be derived from the model which are not properties of the artefact. Mathematical logic (model theory) actually calls such a mathematical construct a 'theory', rather then a 'model'. However, many other disciplines, including engineering, use the term 'model' for these mathematical constructs and this is the meaning adopted in this article. Thus an IDEF0 schema is an 'activity model' of some process, an IDEF1X schema is a 'model of some data', etc.

\section{KNOWLEDGE CATEGORIES}

Knowledge Management (KM) literature defines two main knowledge categories: explicit and tacit. Polanyi (1966) defines tacit knowledge as knowledge, which is implied, but is not actually documented, nevertheless the individual 'knows' it from experience, from other people, or from a combination of sources. Explicit knowledge is externally visible; it is documented tacit knowledge (Junnarkar and Brown, 1997).

Skryme and Amidon (1997) define explicit knowledge as formal, systematic and objective, and it is generally codified in words or numbers. Explicit knowledge can be acquired from a number of sources such as company-internal data, observing business processes, records of policies and procedures, as well as from external sources such as through intelligence gathering. Tacit knowledge is more intangible. 
It resides in an individual's brain and forms the basis on which individuals make decisions and take action, but is not externalised in any form.

Polanyi (1958) also gives another detailed and substantial definition of knowledge categories. He sees tacit knowledge as a personal form of knowledge, which individuals can only obtain from direct experience in a given domain. Tacit knowledge is held in a non-verbal form, and therefore, the holder cannot provide a useful verbal explanation to another individual. Instead, tacit knowledge typically becomes embedded in, for example, routines and cultures. As opposed to this, explicit knowledge can be expressed in symbols and communicated to other individuals by use of these symbols.

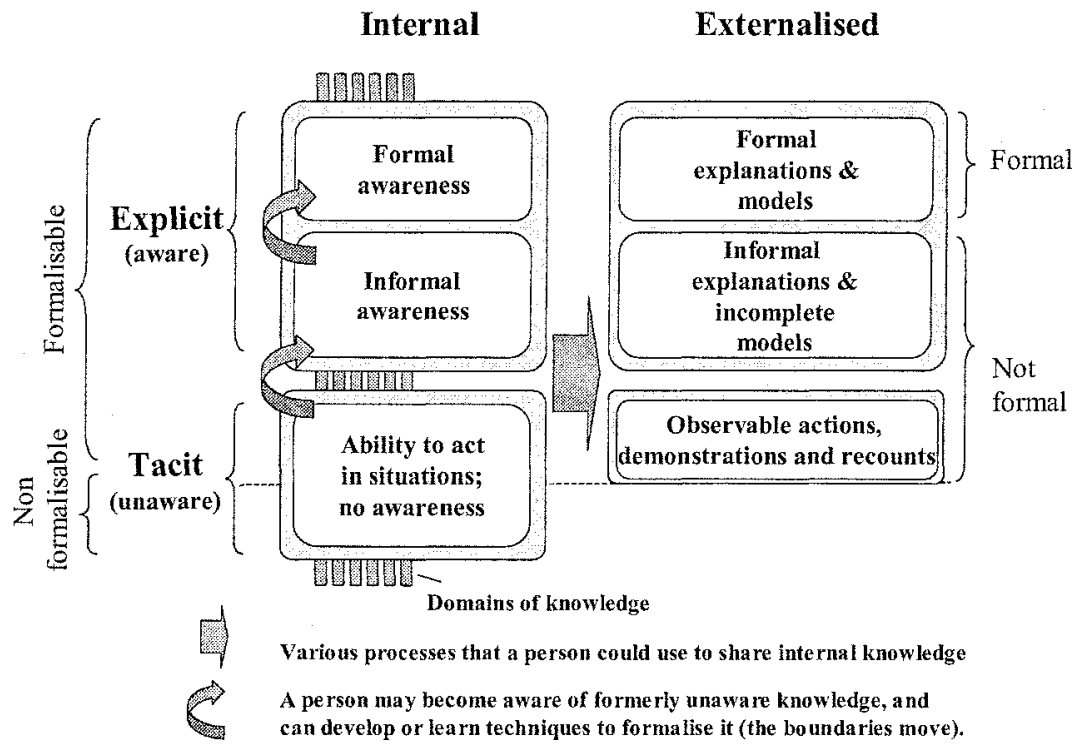

Figure 1. Knowledge categories

Bejierse (1999) states that explicit knowledge is characterised by its ability to be expressed as a word or number, in the form of hard data, scientific formulas, manuals, computer files, documents, patents and standardised procedures or universal works of reference that can easily be transferred and spread. Implicit (tacit) knowledge, on the other hand, is mainly people-bound and difficult to formalise and therefore difficult to transfer or spread. It is mainly located in people's 'hearts and heads'. Considering the above definitions, the authors give the following definitions:

(4) Explicit knowledge is knowledge, which can be articulated and written down. Therefore, such knowledge can (or could) be externalised and consequently shared and disseminated.

(5) Tacit knowledge is subconscious, it is understood and used but it is not identified in a reflective, or aware, way ${ }^{30}$. Tacit knowledge is developed and derives

\footnotetext{
${ }^{30}$ If a person geathers evidence that makes him/her aware of knowledge previously categorised as tacit then this knowledge becomes informal explicit knowledge.
} 
from the practical environment; it is highly pragmatic and often specific to situations in which it has been developed.

Although tacit knowledge is not directly externalisable, it is sometimes possible to create externalisations ${ }^{31}$ that may help someone else to acquire the same tacit knowledge. Tacit knowledge could be made up of insights, judgement, know-how, mental models, intuition and beliefs, and may be shared through direct conversation, telling of stories and sharing common experiences.

Definitions (4) and (5) give rise to a categorisation that can be used to make practically important differentiations between various categories of knowledge. The authors propose to divide knowledge into categories according to the following criteria (see Figure 1):

- Is there awareness of this knowledge explicit/tacit? Awareness means here that the person identifies this knowledge as something he/she is in the possession of and which could potentially be shared with others. In other words, the person not only can use the knowledge to act adequately in situations, but also conceptualises this knowledge. This awareness may be expressed by statements as "I can tell you what to do", "I can explain how to do it". Lack of awareness manifests is statements like "I can not tell you how to do it, but I can show".

- Is the knowledge internalised in a person's head or has it been externalised (internal/externalised)? In other words, have there been any external records made (in form of written text, drawings, models, presentations, demonstrations, etc.)?

- Does the externalisation have a formalised representation or not (formal/notformal)? Formalisation here means that the external representation of the knowledge is in a consistent and complete mathematical/logical form (or equivalent).

Note that each domain of knowledge may contain a mixture of tacit and explicit constituents.

Beside the division of knowledge into aware and unaware categories, additional categorisation of knowledge, according to whether the knowledge could be externalised, into the category of formalisable and non-formalisable, may be added. While explicit knowledge can always be externalised (applying different processes, mechanisms and approaches) tacit knowledge could not be fully externalised, however there are parts that can be communicated through indirect externalised means. This externalisation could be achieved by a) indirect externalisation through conversation, telling of stories, sharing common experiences and other similar approaches, or b) thought an awareness-building process, where the unaware knowledge is transformed into an aware knowledge (even if not formal). A more detailed definition of knowledge processes and their relations to the postulated knowledge categories are presented in Section 3.

\footnotetext{
${ }^{31}$ I.e., these externalisations do not contain a record of the knowledge itself, rather they would contain information that another person could (under certain circumstances) use to construct the same knowedge combining it with his/her already possessed internal knowledge.
} 


\section{KNOWLEDGE PROCESS AND KNOWLEDGE RESOURCES}

A comprehensive survey of the KM literature shows various knowledge management frameworks and KM activities. Some frameworks are composed of very low-level activities and in some frameworks it seems that elementary activities group into higher-level activities.

Nonaka and Takeuchi (1995) define four processes:

- Internalisation is the process in which an individual internalises explicit knowledge to create tacit knowledge. In Fig.1 this corresponds to turning externalised knowledge into internalised - Nonaka does not differentiate between formal and informal awareness.

- Externalisation is the process in which the person turns their tacit knowledge into explicit knowledge through documentation, verbalisation, etc. In Fig. 1 this process corresponds to turning internalised, formalisable knowledge into externalised knowledge and subsequently communicating it (internal $\rightarrow$ externalised).

- Combination is the process where new explicit knowledge is created through the combination of other explicit knowledge.

- Socialisation is the process of transferring tacit knowledge between individuals through observations and working with a mentor or a more skilled / knowledgeable individual. In Fig. 1 this corresponds to tacit knowledge $\rightarrow$ observable actions, etc.

Devenport and Prusak (1998) identify four knowledge processes: knowledge generation (creation and knowledge acquisition), knowledge codification (storing), knowledge transfer (sharing), and knowledge application (these processes can be represented as various transitions between knowledge categories in Figure 1).

Alavi and Marwick (1997) define six KM activities: a) acquisition, b) indexing, c) filtering, d) classification, cataloguing, and integrating, e) distributing, and f) application or knowledge usage, while Holsapple and Whinston (1987) indentfy more comprehensive KM process, composed of the following activities: a) procure, b) organise, c) store, d) maintain, e) analyse, f) create, g) present, h) distribute and i) apply. (Again, these processes can be represented as various transitions between knowledge categories in Figure 1.)

Holsapple and Joshi (2002) present four major categories of knowledge manipulation activities:

- acquiring activity, which identifies knowledge in the external environment (form external sources) and transforms it into a representation that can be internalised and used;

- selecting activity identifying needed knowledge within an organisation's existing resources; this activity is analogous to acquisition, except that it manipulates resources already available in the organisation;

- internalising involves incorporating or making the knowledge part of the organisation, and

- using, which represents an umbrella phrase for a) generation of new knowledge by processing of existing knowledge and b) externalising knowledge that makes knowledge available to the outside of the organisation. 
These four processes are applicable to the organisation as an entity, rather then addressing knowledge processes from the point of view of an individual.

As a conclusion: organisations should be aware of the complete process of knowledge flow, looking at the flow between the organisation and the external world and the flow among individuals within (and outside) the organisation. This latter is an important case, because in many professional organisations individuals belong to various communities, and their links to these communities is equally important to them as the link to their own organisation.

\subsection{Knowledge resources}

Knowledge manipulation activities operate on knowledge resources (KR) to create value for an organisation. On the one hand, value generation depends on the availability and quality of knowledge resource, as well productive use of KR depends on the application of knowledge manipulation skills to execute knowledge manipulation activities.

Holsapple and Joshi (2002) developed a taxonomy of KR, categorising them into schematic and content resources. The taxonomy identifies four schematic resources and two content resources appearing in the form of participant's knowledge and artefacts. Both schema and content are essential parts of an organisation's knowledge resources.

Content knowledge is embodied in usable representations. The primary distinction between participant's knowledge and artefacts lies in the presence or absence of knowledge processing abilities. Participants have knowledge manipulation skills that allow them to process their own repositories of knowledge; artefacts have no such skills. An organisation's participant knowledge is affected by the arrival and departure of participants and by participant learning. As opposed to this, a knowledge artefact does not depend on a participant for its existence. Representing knowledge as an artefact involves embodiment of that knowledge in an object, thus positively affecting its ability to be transferred, shared, and preserved (in Figure 1 knowledge artefacts correspond to recorded externalised knowledge).

Schema knowledge is represented or conveyed in the working of an organisation. It manifests in the organisation's behaviours. Perceptions of schematic knowledge can be captured and embedded in artefacts or in participant's memories, but it exists independent of any participant or artefact. Schematic knowledge resources are interrelated and none can be identified in terms of others. Four schematic knowledge resources could be identified: a) culture (as the basic assumptions and beliefs that are shared by members of an organisation), b) infrastructure (the knowledge about the roles that have been defined for participants), c) purpose (defining an organisation's reason for existence), and d) strategy (defining what to do in order to achieve organisational purpose in an effective manner).

Note, that the above-described content knowledge is also referred to in contemporary management literature and can be named as 'individual knowledge'; while schema knowledge is identified as 'collective knowledge' and is closely related to the organisation's capability.

In addition to its own knowledge resources, an organisation can draw on its environment that holds potential sources of knowledge. Through contacts with its environment, an organisation can replenish its knowledge resources. The 
environmental sources do not actually belong to an organisation nor are they controlled by the organisation. When knowledge is acquired form an environment source, it becomes an organisational source.

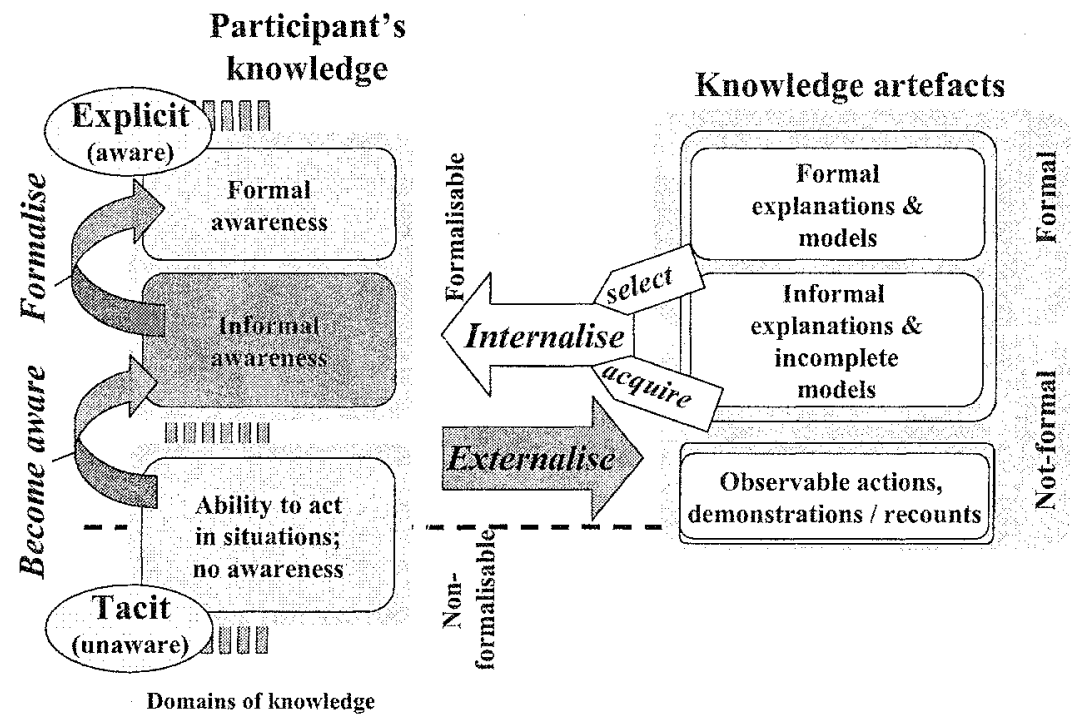

Figure 2. Knowledge process model

\subsection{Knowledge process model}

Considering the definitions of a) knowledge processes proposed by different authors (like Nonaka and Takeuchi (1995), and Holsapple and Joshi (2002)) and b) knowledge categories defined in the knowledge category model in Section 3.2), the authors further propose a knowledge process model, which identifies main internal and external knowledge processes and their relationships to knowledge categories.

This model defines two major categories of knowledge process: the knowledge externalisation process and the knowledge internalisation process.

The knowledge internalisation process, considers the source or environment from where that knowledge derives (originates) and applies two major mechanisms: a) the selection process internalises knowledge from inbound $K R$ and $b$ ) the acquisition process acquires knowledge from external KR. However, a KR could appear in different forms as a) knowledge artefacts in formal or not-formal presentation and b) schema knowledge and knowledge present in data and information which has to be processed (in the form of observation of actions, demonstrations, recount and data and information processing) which is still to be turned into a usable and transferable form of knowledge.

Knowledge externalisation includes the articulation and codification of knowledge in the form of formal or not-formal knowledge. Formal, aware knowledge could be externalised by formal explanations and models, while informal knowledge can be externalised using informal explanations or incomplete models.

Beside the externalisation and internalisation processes, two other important participant-bounded processes can be identified - the awareness process and the formalisation process. The awareness process transforms the formalisable part of 
unaware knowledge into aware knowledge, while the formalisation process converts already aware knowledge into structured and formal form. Awareness and formalisation knowledge processes are discussed in more detail in Section 4.2, where knowledge processes are instantiated according to BPM concepts.

\section{THE ROLE OF MODELS IN KNOWLEDGE MANAGEMENT}

Many knowledge management systems (KMSs) are primarily focused on solutions for the capture, organisation and distribution of knowledge.

Rouggles (1998), for example, found that the four most common KM projects conducted by organisations were creating/implementing an intranet, knowledge repositories, decision support tools, or groupware to support collaboration.

Spender (2002) states that the bulk of KM literature is about computer systems and applications of 'enterprise-wide data collection and collaboration management', which enhance communication volume, timeliness, and precision.

Indeed, current $\mathrm{KM}$ approaches focus too much on techniques and tools that make the captured information available and relatively little attention is paid to those tools and techniques that ensure that the captured information is of high quality or that it can be interpreted in the intended way.

Teece (2002) points out a simple but powerful relationship between the codification of knowledge and the costs of its transfer. Simply stated: the more a given item of knowledge or experience has been codified (formalised in the terminology of Figure 1), the more economically it can be transferred.

Uncodified knowledge is slow and costly to transmit. Ambiguities abound and can be overcome only when communication takes place in face-to-face situations. Errors of interpretation can be corrected by a prompt use of personal feedback.

The transmission of codified knowledge, on the other hand, does not necessarily require face-to-face contact and can often be carried out by mainly impersonal means. Messages are better structured and less ambiguous if they can be transferred in codified form.

Based on the presented features of business process modelling (and in the broader sense enterprise modelling) and the issues in knowledge capturing and shearing, BPM is not only important for process engineering but also as an approach that allows the transformation of informal knowledge into formal knowledge, and that facilitates externalisation, sharing and subsequent knowledge internalisation. BPM has the potential to improve the availability and quality of captured knowledge (due to its formal nature), increase reusability, and consequently reduce the costs of knowledge transfer. The role and contribution of BPM in knowledge management will be discussed in more detail in Section 4.2.

\subsection{BPM and KM are related issues}

While the methods for developing enterprise models have become established during the 1990s (both for business process analysis and design) these methods have concentrated on how such models can support analysis and design teams, and the question of how these models can be used for effective and efficient sharing of information among other stakeholders (such as line managers and engineering practitioners) has been given less attention. 
If enterprise models, such as business process models, embody process knowledge then it must be better understood to what extent and how existing process knowledge can be externalised as formal models, and under what conditions these models may be effectively communicated among stakeholders. Such analysis may reveal why the same model that is perfectly suitable for a business process analyst or designer may not be appropriate for end users in management and engineering. Thus the authors developed a theoretical framework which can give an account of how enterprise models capture and allow the sharing of the knowledge of processes whether they are possessed by individuals or groups of individuals in the company. The framework also helps avoid the raising of false expectations regarding the effects of business modelling efforts.

\subsection{The knowledge life-cycle model}

Figure 3 introduces a simple model of knowledge life-cycle, extending (detailing) the models proposed by Nonaka and Takeuchi (1995), and Zack and Serino (1998). Our extension is based on Bernus et al (1996), which treat enterprise models as objects for semantic interpretation by participants in a conversation, and establishes the criteria for uniform (common) understanding. Understanding is of course most important in knowledge sharing. After all, if a model of company knowledge that can only be interpreted correctly by the person who produced it, is of limited use for anyone else. Moreover, misinterpretation may not always be apparent, thus through the lack of shared interpretation of enterprise models (and lack of guarantees to this effect) may cause damage. This model (Figure 3) represents relations between different types of knowledge, and will be used as a theoretical framework.

In order for employees to be able to execute production, service or decisional processes they must possess some 'working knowledge' (e.g. about process functionality, required process inputs and delivered outputs, organisation, management, etc.). Working knowledge is constantly developed and updated through receiving information from the internal environment (based on the knowledge selection process) and from the external environment (thought the process of knowledge acquisition).

Working knowledge (from the perspective of the knowledge holder) is usually tacit. Knowledge holders don't need to use the possessed knowledge in its explicit, formalised form to support their actions. They simply understand and know what they are doing and how they have to carry out their tasks - having to re-sort to the use of explicit formal knowledge would usually slow down the action.

According to the suitability for formalisation such working knowledge can be divided into two broad groups: formalisable and non-formalisable knowledge. Such division of knowledge into two broad categories seems to closely correspond to how much the process can be structured, i.e. to be decomposed into a set of interrelated lower level constituent processes. These characteristics can be observed when considering knowledge about different typical business process types.

The formalisation and structural description of innovative and creative processes, such as some management, engineering and design processes (or in general the group of ad-hoc processes), is a difficult task, due to the fact that the set of constituent processes is not predefined, nor is the exact nature of their combination well understood by those who have the knowledge. Consequently, knowledge about 
this type of processes could be considered tacit knowledge (because they are not formalisable unaware processes), i.e. not suitable for formalisation/structuring.

In contrast to the characteristics of the group of ad-hoc processes the group of ill-structured and structured (repetitive or algorithmic) processes can be formalised and structured at least to a degree; consequently the knowledge about these processes may become explicit formal knowledge. Examples of such processes are management, engineering and design on the level of co-ordination between activities as performed by separately acting-individuals or groups, and repetitive business and manufacturing activities.

The formalisable part of knowledge (knowledge about structured and illstructured processes) is extremely important and valuable for knowledge management, because this may be distributed and thus shared with relative ease. Namely, the process of transformation of the formalisable part of tacit knowledge into formal knowledge (the formal part of explicit/aware knowledge) represents one of the crucial processes in knowledge management. The authors believe that the cost of knowledge management (measured by the level of reuse and return of investment to the enterprise) in case of formal explicit knowledge would be lower than in case of tacit (unaware) - or even in case of unstructured explicit - knowledge, simply because the sharing of the latter is a slow and involved process.

To be able to perform the aforementioned formalisation process we need additional capabilities known as culturally shared or situation knowledge (e.g. knowledge shared by the community that is expected to uniformly interpret the formal models of the target processes). Culturally shared knowledge plays an essential role in the understanding of the process or entity in question and in its formalisation and structuring. E.g. the definition of an accounting process can only be done by an individual who understands accounting itself, but this formalisation will be interpreted by other individuals who must have an assumed prior culturally shared and situational knowledge that is not part of the formal representation (Bernus et al, 1996).

As mentioned, one of key objectives of KM is the externalisation of participants' knowledge. Regarding the type of knowledge (tacit and explicit) different tools and approaches in knowledge capturing may be used:

- Tacit knowledge (whether formalisable or not) can be transferred through live in situ demonstrations, face-to-face storytelling, or captured informal presentations (e.g. multimedia records, personal accounts of experience, or demonstrations). Note that tacit formalisable knowledge may be discovered through a research process and thus made explicit. Subsequently such knowledge may be captured as described in the bullet point below.

- Explicit knowledge can be captured and presented in external presentations (through the process of knowledge capturing also known as knowledge codification). An external presentation may be formal or not formal. A textual description, like in quality procedure documents (ISO9000) is not formal, while different enterprise models (e.g. functional business process models) are examples of formal external representations of knowledge (knowledge externalisations). 
Formal and informal external representations are called knowledge artefacts. The advantage of using formal models for process description is the quality of the captured knowledge.

To actually formalise knowledge, formalisation skills are needed (in this case business process modelling skills).

The above process of knowledge externalisation has to be complemented by a matching process of knowledge internalisation that is necessary for the use of available knowledge resources.

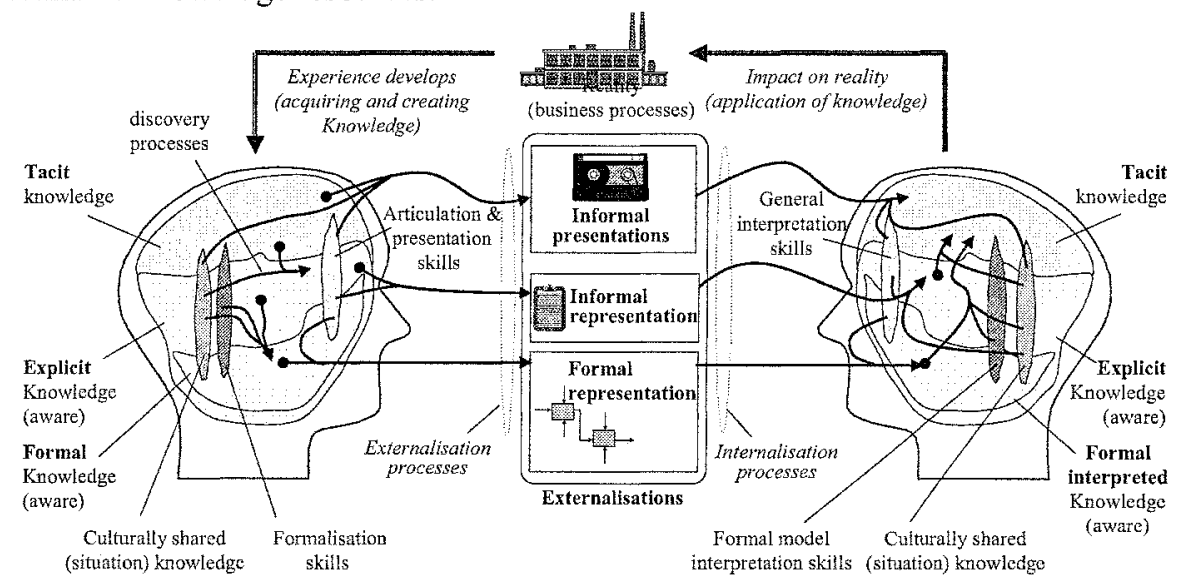

Figure 3: The knowledge life-cycle model

According to the type and form of externalised knowledge, various internalisation processes (and corresponding skills) are necessary. In general, the less formal the presentation / representation, the more prior assumed situation-specific knowledge is necessary for correct interpretation. Conversely, more formal representations allow correct interpretation through the use of more generic knowledge and require less situation-specific knowledge. Thus formalisation helps enlarge the community that can share the given knowledge resource.

An informal external presentation of knowledge accompanied with its interpretation (e.g. interpretation of the presented story) can directly build working (tacit) knowledge, however the use of these presentations is only possible in limited situations, and it is difficult to verify that correct interpretation took place as well as the degree of completeness of such knowledge transfer. However, the verification of correct interpretation and completeness is only possible through direct investigation of the understanding of the individuals who internalised this type of knowledge. This is a serious limitation for knowledge sharing through informal means.

A formal external presentation, such as a business process model developed in the IDEF0 (ICAM DEFinition) modelling languages (Menzel and Mayer, 1998), must be first interpreted to be of use. To interpret the content, i.e. the information captured in this model, knowledge-processing skills (abilities) are needed. Formal model interpretation skills are generic and not situation dependent, therefore even culturally distant groups of people can share them. Still, such formal representation must be further interpreted by reference to culturally shared, prior assumed 
knowledge so that the content of the formal knowledge (information captured in the business process model) can be understood and interpreted in the intended way, and thus integrated into working knowledge (to improve competencies). However, to test for correct interpretability it is possible to test whether the primitive concepts in the model (i.e. those not further explained/decomposed) are commonly understood. If this is the case then the formal nature of the model guarantees uniform interpretability. Completeness can be tested without the direct investigation of the understandings of those individuals who internalise this formal knowledge (i.e. the developer of the formal model can test himself or herself, whether the model is complete - provided the primitive concepts used are uniformly understood ${ }^{32}$ ).

The reuse of formal externalised knowledge could have an impact on the execution of process in terms of their efficiency, according to the well known fact that formally learnt processes must undergo an internalisation process after which they are not used in a step-by-step manner. Therefore, the transfer of the acquired formal knowledge into tacit knowledge is a 'natural' learning process and is necessary for efficiency. The internalisation of externalised formal knowledge thereby closes the loop of the knowledge life-cycle.

Beside the importance of the formalisation/structuring process of knowledge, easy accessibility and distribution of business process models is one of the key factors for a successful deployment of EM practice in organisations. Organisations can use an information infrastructure and a variety of technologies (usually already available and present in organisations) such as an Intranet, web tools, etc., to support storage, indexing, classification, transfer and sharing activities. Using such a distribution mechanism process models can be made available to all stakeholders, and their access can be made platform (software and hardware) independent.

\section{CONCLUSION}

The great interest in Knowledge Management, as one of the hottest research topics of the past decade, is being conditioned by several driving forces: a) recognition of how difficult it is to deal with complexity in the business environment, $b$ ) interest in core competencies, their communication, leverage and possible transfer, c) issues concerning the dissemination of company knowledge in world-wide distributed companies, d) rapid development and adoption of ICT, and e) company awareness of issues concerning individual's knowledge and its externalisation and formalisation.

Companies have already adopted a number of different initiatives, which could become useful components for KM implementation. BPM represents one of these initiatives and a key KM component. BPM as an important tool for KM allows the transformation of informal knowledge into formal knowledge and facilitates its externalisation and sharing.

Beside supporting the knowledge awareness and formalisation process, BPM has the potential to establish the criteria for uniform understanding and improve the availability and quality of captured knowledge (due to its formal nature), increase reusability, and consequently reduce the costs of knowledge transfer.

\footnotetext{
${ }^{32}$ This test is commonly ignored by developers of formal models, probably because they assume that primitive concepts are all known through the users' formal education.
} 
The article developed a further differentiation between various types of knowledge and processes and their mutual relationships (relative to existing knowledge categorisations available in the literature). The proposed knowledge categorisation and definition of key knowledge processes represents the authors' attempt and contribution as a basis for more explicit definitions of key notions in the $\mathrm{KM}$ domain. However, further research should be done to create a unified and widely accepted Knowledge Management ontology.

Because business process models embody process knowledge, a better understanding of the extent and effective communication of business process models must be achieved. Therefore, by use of the presented theoretical framework this article gave an account of how enterprise models capture and allow the sharing of the knowledge encapsulated in processes. The framework also:

- helps to avoid the raising of unrealistic expectations regarding the effects of business modelling efforts

- presents major knowledge categories, stages in knowledge transformation and activities in this process

- defines the correlation between the formalisable and non-formalisable knowledge categories and process types and

- emphasises the importance of the transformation process on the formalisable part of the knowledge, into its formal presentation as one of the crucial processes in knowledge management.

\section{REFERENCES}

Alavi, M., Marwick, P. (1997) One Giant Brain. Boston (MA) : Harvard Business School. Case 9-397-108

Baker M., Baker, M., Thorne, J., Dutnell, M. (1997) Leveraging Human Capital. Journal of Knowledge Management. MCB University Press. 01:1 pp63-74

Beijerese, R.P. (1999) Questions in knowledge management: defining and conceptualising a phenomenon. Journal of Knowledge Management. MCB University Press. 03:2 pp94-110

Bennet, D., Bennet, A. (2002) The Rise of the Knowledge Organisations. in Holsapple, C.W. (Eds.) Handbook on Knowledge Management 1. Berlin : Springer-Verlag. pp5-20

Bender, S., Fish, A. (2000) The transfer of knowledge and the retention of expertise: the continuing need for global assignments. Journal of Knowledge Management. MCB University Pres. 04:2 pp 125-137

Bernus P., Nemes, L., Moriss, B. (1996) The Meaning of an Enterprise Model. in Bernus, P., Nemes, L. (Eds.) Modelling and Methodologies for Enterprise Integration. London : Chapman and Hall. pp183-200

Chen, D., Doumeingts, G. (1996) The GRAI-GIM reference model, architecture and methodology. in Bernus, P., Nemes, L. and Williams, T.J. (Eds.) Architectures for Enterprise Integration. London : Chapman \& Hall. pp 102-126

Conner, K., Prahalad, C.K. (1996) A resource-based theory of the firm: Knowledge versus opportunism. Organization Science. Vol. 7 pp477-501

Davenport, T.H. (1993) Process innovation: reengineering work through information technology. Boston (MA) : Harward Business School Press 
Davenport, T. H., Prusak, L. (1998) Working Knowledge: How Organizations

Manage What They Know. Boston (MA) : Harvard Business School Press. pp16

Holsapple, C.W., Joshi, K.D. (2002) A Knowledge Management Ontology. in

Holsapple, C.W. (Eds.) Handbook on Knowledge Management 1, Berlin :

Springer-Verlag. pp89-128

Holsapple, C.W., Whinston., A.B. (1987) "Knowledge-based Organizations."

Information Society. (2) pp 77-89

ISO/TC 176/SC2 (2000) ISO9004:2000 Quality management systems - guidelines

for performance improvements

Junnarkar, B., Brown, C.V. (1997) Re-assessing the Enabling Role of Information

Technology in KM. Journal of Knowledge Management. MCB University Press.

01:2 pp 142-148

Menzel, C., Mayer, R.J. (1998) The IDEF family of Languages, in: Bernus, P.,

Nemes, L. and Williams, T.J. (Eds.) Architectures for Enterprise Integration.

London : Chapman \& Hall. pp102-126

Nonaka, I., Takeuchi, H. (1995) The Knowledge - Creating Company: How

Japanese Companies Create the Dynamics of Innovation. New York : Oxford

University Press

Oxford University Press (1999) The Oxford English dictionary. Version 2.0

Polanyi, M. (1958) Personal Knowledge. University of Chicago Press

Polanyi, M. (1966) Tacit Dimension. New York : Doubleday

Rouggles, R. (1998) The State of the Notion: Knowledge Management in Practice.

California Management Review. 40(3) pp80-89

Schultze, U. (2002) On Knowledge Work. in: Holsapple, C.W. (Eds.) Handbook on

Knowledge Management 1, Berlin : Springer-Verlag. pp43-58

Skyrme, D., Amidon, D. (1997) The Knowledge Agenda. Journal of Knowledge

Management, MCB University Press. 01:1 pp27-37

Spender, J.C. (2002) Knowledge Fields: Some Post-9/11 Thoughts about the

Knowledge-Based Theory of the Firm. in: Holsapple, C.W. (Eds.) Handbook on

Knowledge Management 1. Berlin: Springer-Verlag. pp59-72

Teece, D.J. (2002) Knowledge and Competence as Strategic Assets. in: Holsapple,

C.W. (Eds.) Handbook on Knowledge Management 1. Berlin : Springer-Verlag. pp 129-152

Vernadat, F. (1996) Enterprise Modelling and Integration - Principles and

Applications. Chapman \& Hall

Vernadat, F. (1998) The CIMOSA Languages. in: Bernus, P., Mertins, K. and

Schmidt G. (Eds.) Handbook on Architectures of Information Systems. Berlin :

Springer - Verlag. pp243-264

Zack, M.H., Serino, M. (1998) Knowledge Management and Collaboration

Technologies. Lotus Development Corporation. 